\title{
Does the external remote controller's reading correspond to the actual lengthening in magnetic-controlled growing rods?
}

\author{
Kar Hao Teoh ${ }^{1,2}$ - $\cdot$ Abdul Nazeer Moideen ${ }^{1,2} \cdot$ Kausik Mukherjee $^{3} \cdot$ Sridhar Kamath $^{3} \cdot$ Stuart H. James $^{2}$. \\ Alwyn Jones $^{2} \cdot$ John Howes ${ }^{2} \cdot$ Paul R. Davies ${ }^{2} \cdot$ Sashin Ahuja ${ }^{1,2}$
}

Received: 31 July 2019 / Revised: 12 January 2020 / Accepted: 29 January 2020 / Published online: 25 February 2020

(c) The Author(s) 2020

\begin{abstract}
Purpose Magnetic-controlled growing rods (MCGRs) are now routinely used in many centres to treat early-onset scoliosis (EOS). MCGR lengthening is done non-invasively by the external remote controller (ERC). Our experience suggests that there may be a discrepancy between the reported rod lengthening on the ERC and the actual rod lengthening. The aim of this study was to investigate this discrepancy.

Methods This was a prospective series. Eleven patients who were already undergoing treatment for EOS using MCGRs were included in this study.

Results One hundred and ninety-two sets of ultrasound readings were obtained (96 episodes of rod lengthening on dual-rod constructs) and compared to their ERC readings. Only 15/192 (7.8\%) readings were accurate; 27 readings (14.9\%) were false positive; and 8 readings (4.2\%) were an underestimation while 142 readings $(74.0 \%)$ were an overestimation by the ERC. Average over-reporting by the ERC was 5.31 times of the actual/ultrasound reading. When comparing interval radiographs with lengthening obtained on ultrasound, there was a discrepancy with an average overestimation of 1.35 times with ultrasound in our series. There was a significant difference between ERC and USS $(p=0.01)$ and ERC and XR $(p=0.001)$. However, there was no significant difference between USS and XR $(p>0.99)$.

Conclusion The reading on the ERC does not equate to the actual rod lengthening. The authors would recommend that clinicians using the MCGR for the treatment of early-onset scoliosis include pre- and post-extension imaging (radiographs or ultrasound) to confirm extension lengths at each outpatient extension. In centres with ultrasound facilities, we would suggest that patients should have ultrasound to monitor each lengthening after distraction but also 6-month radiographs.
\end{abstract}

\section{Graphic abstract}

These slides can be retrieved under Electronic Supplementary Material.
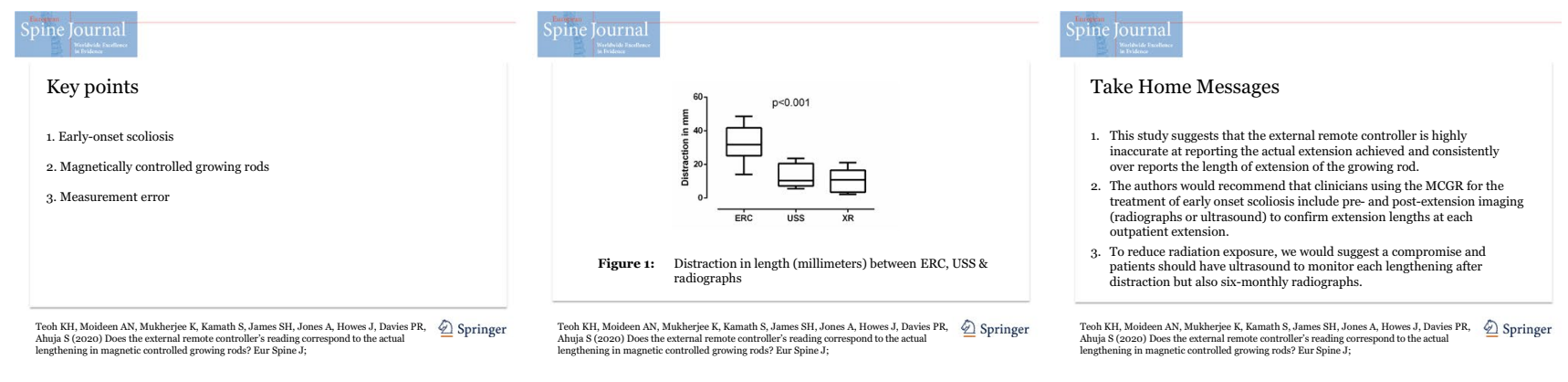

Electronic supplementary material The online version of this article (https://doi.org/10.1007/s00586-020-06335-5) contains supplementary material, which is available to authorized users.

Extended author information available on the last page of the article 
Keywords Early-onset scoliosis · Magnetic-controlled growing rods $\cdot$ Ultrasound $\cdot$ Measurement error $\cdot$ External remote controller

\section{Introduction}

Early-onset scoliosis (EOS) is an abnormal curvature of the spine diagnosed before the age of ten. The best treatment for EOS is unknown, with options ranging from bracing to surgery. Following failure of non-operative treatment, surgical treatment is usually the only option as these curves are generally progressive. Growth sparing spinal surgery is usually preferred as it allows correction of the curve while maintaining growth of the spinal column until the child is close to skeletal maturity when a final spinal fusion operation can be performed.

In June 2014, the National Institute for Health and Care Excellence (NICE) in the UK approved the use of magneticcontrolled growing rods (MCGRs) in EOS on the basis of efficacy and cost-effectiveness [1]. Prior to the introduction of magnetic-controlled growing rods (MCGRs), one of the main options for growth sparing spinal surgery was conventional growing rods. Conventional growing rods normally require 6-month operations to lengthen the rods under general anaesthesia throughout childhood and is associated with high complication rates, e.g. wound infections [2, 3].

However, in MCGR, non-invasive spinal lengthening is performed in the outpatient clinic without sedation or anaesthesia. The magnet within the actuator in MCGR is connected to a lead screw that can be rotated non-invasively by a magnetic field with a handheld device known as external remote controller (ERC), which also contains a permanent magnet. The amount of lengthening achieved is displayed on the ERC device in millimetres. The amount of lengthening can also be assessed radiologically using serial radiographs, fluoroscopy or ultrasound.

Radiograph is the gold standard for assessing the amount of lengthening obtained following a non-invasive spinal lengthening. As there is a radiation risk associated with radiographs, they are not always used after each distraction in some centres. This is particularly so for centres who perform more frequent distractions. At the moment, the optimal frequency of distractions and the amount of lengthening done at each outpatient appointment remain uncertain and this practice is evolving in many centres. There is an increasing trend towards the use of ultrasound as a method of assessing the amount of lengthening because there is no risk of radiation exposure. Ultrasound has previously been validated by two studies as a reliable method of measuring distractions with gold standard radiographs as controls [4, 5]. In our centre, distractions are carried out at 8-10 weekly intervals. Since 2014, we started using ultrasound in our centre in conjunction with our unit's radiologists. We also took yearly interval radiographs as a routine in our centre. The experience within our unit suggests that there may be a discrepancy between the reported rod lengthening on the ERC and the actual rod lengthening when using the MCGR system. The aim of this study was to determine whether a genuine discrepancy exists between the reported rod lengthening on the ERC and the actual rod lengthening on both ultrasound and interval radiographs.

\section{Methods}

\section{Study design and patients}

This was a prospective series comparing rod length measurements using ultrasound and the reported lengthening obtained on the external remote controller (ERC). The study was conducted between February 2014 and April 2018. Over this time period, patients also had radiographs on a yearly basis to ensure that the construct was intact. Eleven patients who were already undergoing treatment for EOS using MCGRs were included in this study. The average age of the patients was 8.9 years (range: 4.5 years to 14 years). All eleven patients had dual-rod constructs. There were eight males and three females. Seven were primary procedures, while four were revisions from conventional growing rod system. Table 1 shows the demographic of our study cohort.

\section{Surgical procedure and our follow-up protocol}

The MCGR system used was the MAGEC, MAGnetic Expansion Control (Ellipse Technologies Inc, Irvine, California, USA). This comprises one or two sterile titanium implantable growth rods with a magnet in the actuator that drives the lengthening process magnetically. The diameter of the rods used depended on the child's body weight, and the choice of a single- or a dual-rod construct was down to surgeon's preference.

Under general anaesthesia, patients were positioned prone, with intravenous antibiotics given on induction. The revision procedures were performed through a standard open posterior midline approach with insertion of pedicle screws or lamina hooks proximally and distally to the curve. However, the primary procedures were performed with two separate incisions, one proximal and one distal to the curve for the anchors. The length of the MAGEC rod was cut to fit the patient and contoured. The MAGEC rod 
was then connected to the proximal and distal anchorages. For the primary procedures, the rods were rail-road and inserted through the submuscular layer. The diameter of the MAGEC rods used in our series was all $5.5 \mathrm{~mm}$.

After surgery, patients were followed up in clinic at 6 weeks initially. Non-invasive distraction of the MAGEC rods was started between 3 and 6 months from the initial implantation. In our centre, distractions are carried out at 8-10 weekly intervals. During each visit, patients were positioned prone. Using a handheld magnet wand, the magnet in the actuator was located, and this site was marked. A handheld magnetic ERC was placed over this marking to lengthen the rod, thus distracting the spine. The distraction/lengthening was measured as per the display on the ERC and recorded in millimetres. This equates to the intended distraction (ID).

\section{Measurement of lengthening using ultrasound}

Ultrasound imaging was performed on the magnetic rods both pre- and post-lengthening by two consultant radiologists with patients in the prone position using a Toshiba Aplio 500 ultrasound machine utilizing an 12-10 MHz linear ultrasound transducer and standard coupling gel. Measurements were made from the point at which the extendible part of the rod enters the actuator housing to the first tapering point of the rod as this was consistently identifiable (Figs. 1, 2). Three separate ultrasound measurements were performed, and the final reading was logged as the average
Table 1 Patient demographics of our study cohort
Fig. 1 Pre-extension ultrasound image measuring the distance between the actuator housing and the taper of rod, in this case measuring $34.2 \mathrm{~mm}$

\begin{tabular}{|c|c|c|c|c|c|c|}
\hline Patient & $\begin{array}{l}\text { Age at } \\
\text { operation } \\
\text { (years) }\end{array}$ & Sex & Curve & Primary or revision & Diagnosis & Previous surgery \\
\hline 1 & 5 & $\mathrm{~F}$ & Right & Primary & Idiopathic & \\
\hline 2 & 8 & M & Right & Primary & Idiopathic & \\
\hline 3 & 4 & M & Right & Primary & Idiopathic & \\
\hline 4 & 9 & $\mathrm{~F}$ & Right & Revision & Idiopathic & Expedium to Magec 2015 \\
\hline 5 & 5 & M & Left & Primary & Neuromuscular & \\
\hline 6 & 11 & M & Left & Revision & Idiopathic & Expedium to Magec 2014 \\
\hline 7 & 9 & M & Right & Revision & Idiopathic & Expedium to Magec 2011 \\
\hline 8 & 6 & $\mathrm{~F}$ & Right & Primary & Idiopathic & \\
\hline 9 & 6 & M & Right & Primary & Idiopathic & \\
\hline 10 & 5 & M & Left & Primary & Congential & \\
\hline 11 & 10 & M & Left & Revision & Syndromic & ISOLA to Magec 2011 \\
\hline
\end{tabular}

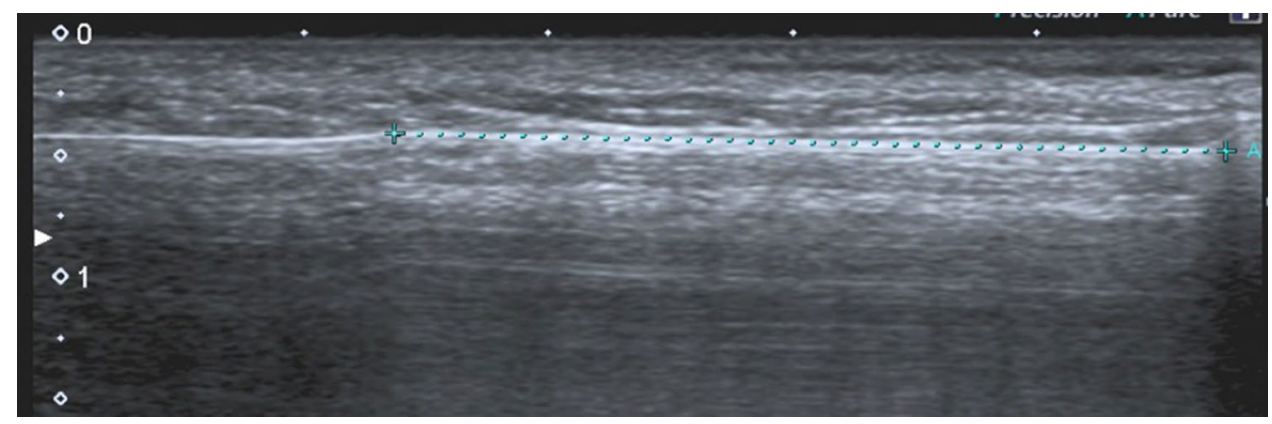

Fig. 2 Post-extension ultrasound image measuring $36.7 \mathrm{~mm}$. Total extension achieved $=2.5 \mathrm{~mm}$

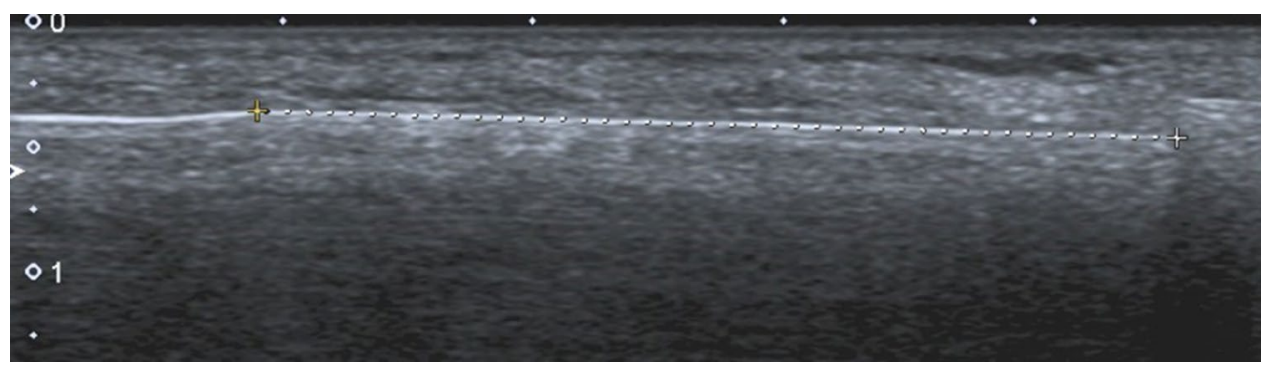


of the three readings as per the best view on ultrasound to assess the distraction of the magnet. This was recorded in millimetres. As a practice, if ultrasound measurements did not show any lengthening, a radiograph was obtained to make sure the rod and the anchoring metalwork were intact.

\section{Measurement of lengthening using radiographs}

Whole spine posterioanterior (PA) radiographs were taken with the patient in the erect position. Images were uploaded to a picture archiving and communication system (PACS). Rod length measurements were performed by a single spinal surgeon using the inbuilt PACS measuring tool. Calibration of the images was performed by measuring the diameter of the actuator portion of the MAGEC rod and multiplying it by the actual diameter which is $9.02 \mathrm{~mm}$ as per the surgical technique from the manufacturer. The distraction length was measured by measuring the consistent radiographic feature in the rod which appears as a 'window' in the centre portion of the actuator. This is multiplied by the calibration to obtain the true distraction length.

\section{Data collection and statistical analysis}

The lengthening achieved on ultrasound scan was compared to the reading recorded on the ERC. The lengthening achieved on ultrasound scan was divided by the ERC reading and expressed as a percentage. A percentage of $>105 \%$ indicates an underestimation of the actual rod lengthening by the ERC, while a percentage of $<95 \%$ indicates an overestimation by the ERC. Accounting for a 5\% standard error, an accurate reading was taken as $100 \pm 5 \%$. The lengthening obtained between the interval radiographs was measured and compared to the cumulative ERC readings and cumulative ultrasound readings over the same time period for each patient. If there was metalwork failure, we took the last available normal radiograph to ensure it was a fair comparison.

Data were analysed using SPSS Statistics software 20.0 (IBM, Armonk, NY, USA). Analysis of variance was used to compare between groups.

\section{Results}

In total, 96 non-invasive rod lengthenings were undertaken on 11 patients with dual-rod constructs over this time period. This provided 192 sets of readings.

\section{ERC versus ultrasound}

Only 15/192 (7.8\%) readings were accurate. Twenty-seven readings (14.0\%) showed no lengthening on ultrasound but the ERC showed lengthening (false positive). In eight readings (4.2\%), there was an underestimation of the actual rod lengthening by the ERC. In 142 readings (74.0\%), there was an overestimation of the actual rod lengthening by the ERC, with a mean actual lengthening of $36 \%$ from the reported lengthening. Average over-reporting by ERC was 5.31 times of the actual/ultrasound reading (range 1-40). There was significant statistical difference between ERC versus USS $(p=0.01)$. There was no significant statistical difference between distraction obtained between left and right rods ( $p=0.67$, Wilcoxon signed-rank test). There were also no statistical differences found between primary and revision cases, age of onset, BMI, side of curve, idiopathic versus non-idiopathic and pre-surgical magnitude $(p>0.05)$.

\section{ERC Versus Ultrasound Versus Radiographs}

The cumulative ERC, ultrasound and radiographic lengthening were calculated over the time period of the interval radiographs. Three patients were excluded here as they did not have a full year of data. In our study, the ERC consistently overestimates the lengthening on both USS and radiographs (Fig. 3). Using radiographs as the gold standard, there is still a discrepancy with ultrasound with an average overestimation of 1.35 times (range: $0.92-3.1$ ) in our series. There were significant differences between the readings for ERC, ultrasound and radiographs at the yearly interval $(p<0.001)$.

\section{Discussion}

Our study indicates that only $8 \%$ of the ERC readings were accurate, while $74 \%$ of the ERC reading overestimated the actual rod lengthening. Previous studies have focused on assessing the reliability of using ultrasound rather than radiographs for assessing distraction in MCGR [4, 5]. A recent study has shown somewhat similar findings to ours [6]. They concluded that ultrasound can provide confirmatory information of non-invasive lengthening of MCGR but they tend to

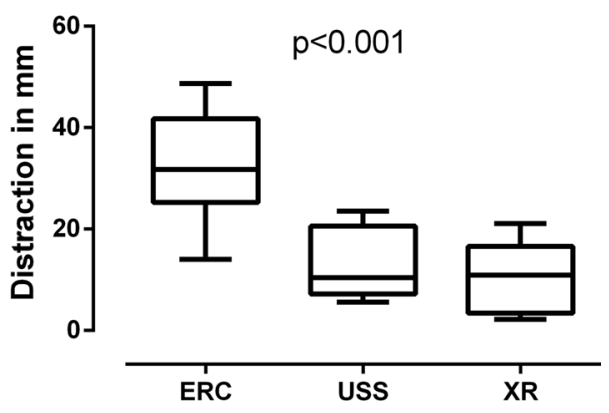

Fig. 3 Distraction in length (millimetres) between ERC, USS and radiographs 
underestimate the achieved length as measured by radiographs only in primary cases. In their study, they found that revision cases demonstrate better concordance between ultrasound and radiographs. This was not observed in our study and could be due to a higher number of revision cases in their study. In our series, we found that ultrasound tends to overestimate the achieved length as measured by radiographs. The reason for this could be due to the difference in frequency of the radiograph taken in our series (3 months vs. yearly). As our unit adopted an ultrasound approach to confirming distraction based on other validation studies $[4,5]$, therefore we did not take radiographs every distraction like their study.

A prospective case series of magnetic-controlled growing rods in 2012 by Cheung et al. [7] reported an actual lengthening of the growing rods of $78.9 \%$ of the predicted lengthening (or 1.28 times more of the actual lengthening) when comparing pre- and post-lengthening on plain radiograph. The case series, which consisted of only two patients with three rods but with a total of 45 extensions, reported a good relationship between the predicted and actual distraction lengths and no difficulty with monthly distractions. In contrast, our study demonstrates 27 occasions (14\%) when no distraction was achieved despite the ERC reporting a positive lengthening. The discrepancy between our results and the results of Cheung et al. is likely to be due to a difference in the number of patients and growing rods in the two studies. A large retrospective, consecutive, multicentre case series of 30 MCGR patients with a mean follow-up of 18 months reported that there was a $45.5 \%$ discrepancy between their intended total distraction and their total measured distraction on radiographs [8].

A recent study reported similar findings when they compared the ERC readings with fluoroscopy. In their study of 21 patients, true distraction (TD) was determined by measuring the expansion gap on dedicated fluoroscopic images of the actuator and this was compared to the intended distraction (ID) reported on the ERC. The true-to-intended distraction ratio was calculated as 0.33 , and the difference between the ID and true TD varied from 2 to $44 \mathrm{~mm}$ [9]. In the follow-up study, Ahmad et al. have also shown a difference between true distraction (T) and intended distraction (I) in their series of 35 patients followed over 57 months treated with MCGRs. Patients were distracted at 3-month intervals and had fluoroscopic images taken immediately after the distraction. There was also a significant decrease in T/I ratio which was a gradual linear decline over time [10].

The traditional method of measuring the lengthening of MCGRs is using plain radiographs [6]. The use of ultrasound imaging is associated with no exposure to ionizing radiation for patients with MCGR. A previous study demonstrated that for every set of pre-lengthening and post-lengthening radiographs, MCGR patients are exposed to a mean effective radiation dose of $0.26 \mathrm{mSv}$ and an additional mean attributable lifetime cancer risk of approximately 1 in 39,686 [5].
Radiation exposure in children is associated with radiationinduced cancer and as few as 25 whole spine radiographs have been reported to increase the risk of breast cancer by $70 \%$ in females [11]. Two previous studies have shown that distraction of the MCGR noted on radiographs can be reproduced on ultrasound with high inter-rater reliability and intra-rater reliability. Yoon et al. [5] reported an intra-rater reliability of 0.983 (95\% confidence interval $0.956,1.000)$ and an interrater reliability of 0.987 (95\% confidence interval 0.966 , 1.000 ) between ultrasound and radiograph for rod length. Stokes et al. [4] reported that when a rod showed distraction on X-rays of $2 \mathrm{~mm}$, the mean measurement on ultrasound was $1.7 \mathrm{~mm}$ (SD: $0.24 \mathrm{~mm}$ ) while in scenarios where no distraction of the rod was noted on plain radiographs, the ultrasound measurements showed no significant difference. A paper by Cheung et al. [12] which looked at the learning curve in monitoring MCGR distractions with ultrasound found that correlation between radiograph and ultrasound measurements is reasonable to begin with but improves with time. In our series, all our measurements were made by two consultant musculoskeletal radiologists who are highly experienced, and we do not feel this would have played a factor in our results.

Schiedel et al. [13] reported similar problems with magnetic-controlled growing rods used as an intramedullary device in children for limb lengthening. In addition to reporting that $2 / 26$ nails failed to extend with the use of an ERC, they also report a discrepancy between planned extension lengths and achieved length. The largest difference being $8 \mathrm{~mm}$ of achieved lengthening compared with $23 \mathrm{~mm}$ of planned extension, and they found ERC usage was problematic mostly in patients with femoral lengthening.

Although we adopted ultrasound to measure lengthening like many centres, we also continued with radiographs but done at yearly intervals. The reason for this is twofold: to ensure that metalwork is intact and to measure interval lengthening. One weakness of this study was we might not have detected an issue with lengthening at a particular distraction as we only did the radiographs yearly. However, if no lengthening is obtained on ultrasound, it was our department protocol to radiograph the child to ensure the metalwork is intact.

The National Institute for Health and Clinical Excellence (NICE) is a non-departmental public body of the Department of Health in the UK which makes evidence-based recommendations developed by independent committees, including professionals and lay members and consulted on by stakeholders. These appraisals are based primarily on evaluations of efficacy and cost-effectiveness. Section 3.27 of the NICE guidance for the use of MAGECTM growing rods states that 'the committee considered the potential impact of additional imaging associated with more frequent distractions. The committee was advised that X-rays were not always used after each distraction and that ultrasound may be an option for more frequent distractions' [1]. 
Although there is now enough evidence in the literature to use ultrasound as the imaging of choice, we would like to draw caution to this. The ERC is wholly inaccurate, and the readings should be disregarded as reported in many studies. Our results suggest that radiographs still remain the gold standard. We would suggest that patients should have ultrasound to monitor each lengthening after distraction but also 6-month radiographs. This would be no different in patients who have been treated conservatively in brace or being extended 6 months for conventional growing rods.

\section{Conclusion}

This study suggests that the external remote controller is highly inaccurate at reporting the actual extension achieved. The ERC consistently over-reports the length of extension of the growing rod. The authors would therefore recommend that clinicians using the MCGR for the treatment of early-onset scoliosis include pre- and post-extension imaging (radiographs or ultrasound) to confirm extension lengths at each outpatient extension. To reduce radiation exposure, we would suggest a compromise and patients should have ultrasound to monitor each lengthening after distraction but also 6-month radiographs.

\section{Compliance with ethical standards}

Conflict of interest There is no conflict of interest with related to this work.

Disclosures KHT, ANM, KM, SK: Nothing to disclose. SHJ: Other: K2M (Educational contract), NuVasive (Educational contract), outside the submitted work. AJ: Other: NuVasive (Teaching contract), outside the submitted work. JH: Other: Alphatec (Educational contract), outside the submitted work. PRD: Other: K2M (Educational meetings), Globus (Educational meetings), outside the submitted work. SA: Other: Depuy Synthes UK Ltd (Fund a fellowship for the unit), NuVasive UK Ltd (Fund a fellowship for the unit); Personal Fees: Globus Medical (Consulting fee for conven- ing a course in 2013 and 2015), Medtronic (Consulting fee in 2015), outside the submitted work.

Open Access This article is licensed under a Creative Commons Attribution 4.0 International License, which permits use, sharing, adaptation, distribution and reproduction in any medium or format, as long as you give appropriate credit to the original author(s) and the source, provide a link to the Creative Commons licence, and indicate if changes were made. The images or other third party material in this article are included in the article's Creative Commons licence, unless indicated otherwise in a credit line to the material. If material is not included in the article's Creative Commons licence and your intended use is not permitted by statutory regulation or exceeds the permitted use, you will need to obtain permission directly from the copyright holder. To view a copy of this licence, visit http://creativecommons.org/licenses/by/4.0/.

\section{References}

1. National Institute for Health and Care Excellence (June 2014) The MAGEC System for spinal lengthening in children with scoliosis. Medical Technological Guidance MTG18. NICE, London

2. Teoh KH, Winson DM, James SH, Jones A, Howes J, Davies PR, Ahuja S (2016) Do magnetic growing rods have lower complication rates compared with conventional growing rods? Spine J 16(4):S40-S44

3. Bess S, Akbarnia BA, Thompson GH et al (2010) Complications of growing-rod treatmentfor early-onset scoliosis: analysis of one hundred and forty patients. J Bone Joint Surg Am 92-A:2533-2543

4. Stokes OM, O'Donovan EJ, Samartzis D, Luk KDK, Cheung KM-C (2014) Reducing radiation exposure in early-onset scoliosis surgery patients: novel use of ultrasonography to measure lengthening in magnetically-controlled growing rods. Spine $\mathbf{J}$ 14:2397-2404

5. Yoon WW, Chang AC, Tyler P, Butt S, Raniga S, Noordeen H (2015) The use of ultrasound in comparison to radiography in magnetically controlled growth rod lengthening measurement: a prospective study. Eur Spine J 24(7):1422-1426

6. Cobanoglu M, Shah SA, Gabos P et al (2019) Comparison of intended lengthening of magnetically controlled growing rods: ultrasound versus X-ray. J Pediatr Orthop 39(2):e141-e146

7. Cheung KM-C, Cheung JP-Y, Samartzis D, Mak K-C, Wong Y-W, Cheung W-Y, Akbarnia BA, Luk KD-K (2012) Magnetically controlled growing rods for severe spinal curvature in young children: a prospective case series. Lancet 379:1967-1974

8. Lebon J, Batailler C, Wargny M et al (2017) Magnetically controlled growing rod in early onset scoliosis: a 30-case multicenter study. Eur Spine J 26:1567-1576

9. Rolton D, Thakar C, Wilson-MacDonald J, Nnadi C (2016) Radiological and clinical assessment of the distraction achieved with remotely expandable growing rods in early onset scoliosis. Eur Spine J 25(10):3371-3376

10. Ahmad A, Subramanian T, Panteliadis P, Wilson-Macdonald J, Rothenfluh DA, Nnadi C (2017) Quantifying the 'law of diminishing returns' in magnetically controlled growing rods. Bone Joint J 99-B:1658-1664

11. Doody MM, Lonstein JE, Stovall M et al (2000) Breast cancer mortality after diagnostic radiography: findings from the U.S. Scoliosis Cohort Study. Spine 25:2052-2063

12. Cheung JP, Yiu KK, Bow C, Cheung PW, Samartzis D, Cheung KM (2017) Learning curve in monitoring magnetically controlled growing rod distractions with ultrasound. Spine (Phila Pa 1976) 42(17):1289-1294

13. Schiedel FM, Voqt B, Tretow HL, Schuhknecht B, Gosheger G, Horter MJ, Rodl R (2014) How precise if the PRECICE compared to the ISKD in intramedullary limb lengthening? Reliabilty and safety in 26 procedures. ActaOrhop 85(3):293-298

Publisher's Note Springer Nature remains neutral with regard to jurisdictional claims in published maps and institutional affiliations. 


\section{Affiliations}

Kar Hao Teoh ${ }^{1,2}\left(\mathbb{D} \cdot\right.$ Abdul Nazeer Moideen $^{1,2} \cdot$ Kausik Mukherjee $^{3} \cdot$ Sridhar Kamath $^{3}$. Stuart H. James ${ }^{2}$. Alwyn Jones $^{2} \cdot$ John Howes ${ }^{2}$ P Paul R. Davies ${ }^{2}$. Sashin Ahuja ${ }^{1,2}$

Kar Hao Teoh

teohkh@cardiff.ac.uk; karhao@gmail.com

1 School of Engineering, Cardiff University, Queen's Buildings, The Parade, Cardiff CF24 3AA, UK
Welsh Centre for Spinal Surgery and Trauma, University Hospital of Wales, Heath Park, Cardiff CF14 4XW, UK

3 Department of Radiology, University Hospital of Wales, Heath Park, Cardiff CF14 4XW, UK 